\title{
ARTICLE
}

\section{A revised Jenkins formula for electron induced neutron deep penetration calculation}

\author{
Hiroshi Iwase*, Yoshihito Namito and Hideo Hirayama
}

KEK, 1-1 Oho, Tsukuba, Ibaraki 305-0801, Japan

\begin{abstract}
In electron accelerator facility, neutron production induced by high energy electron above $1 \mathrm{GeV}$ hitting on thick target, and the neutron deep penetration for concrete shielding can be calculated by the Jenkins formula. In the formula, the neutron source term is divided into three energy regions of GR, MID, and HE, and neutron deep penetration is solved by summing up the attenuation of the three individual source terms with different attenuation length. In this study, the concept of Jenkins formula is verified by using recent Monte Carlo codes with evaluated data and cross sections, and some parameters of Jenkins formula are revised.
\end{abstract}

\section{Keywords: neutron shielding; electron accelerator; Monte Carlo; PHITS ; deep penetration in concrete}

\section{Introduction}

Radiation calculation using semi-empirical formula has been widely and long period used for variety of purposes, such as, determine radiation yields and dose, design accelerator facilities, and so on. On the other hand, recent highly developed Monte Carlo codes became very powerful method to evaluate complicated radiations (variety of particles and energies) and geometrical conditions with quite precise accuracy. Semi-empirical formula, however, can be still the most important tool in radiation calculation, since it does not take time to obtain an order of correct result without human error, in contrast it is surely not easy to perform an order estimation promptly by Monte Carlo calculations, even by experts. In this study, we focused the electron induced neutron deep penetration in concrete calculation formula by Jenkins [1], and verify the detail of the formula using recent Monte Carlo codes and data.

\section{Methods}

First the original Jenkins formula is shortly described in Sec. 2.1. Then the SLAC experiment [2], which becomes the benchmark data of this study, was reanalyzed concerning the neutron detector response in Sec. 2.2. Finally, some parameters of the original Jenkins formula are re-evaluated by the new benchmark data with results of a Monte Carlo simulations.

In this study, Monte Carlo calculations are performed using the PHITS code [3] together with the JENDL-HE2007[4] evaluated data library.

*Corresponding author. Email: hiroshi.Iwase@kek.jp

\subsection{Jenkins formula}

\subsubsection{Concept and formula}

High energy electron induced neutron dose deep penetration calculation formula was described by Jenkins et al. [1]. The formula was developed based on a benchmark experimental data carried out at SLAC [2] with the assumption that the measured neutron dose attenuation curve is constructed by three different gradients, i.e., three attenuation length of different energy groups of GR (Giant resonance neutrons, E $<20$ $\mathrm{MeV}$ ), MID (Mid-energy neutrons, $20 \mathrm{MeV}<\mathrm{E}<100$ $\mathrm{MeV}$ ) and $\mathrm{HE}$ (High energy neutrons, $100 \mathrm{MeV}<\mathrm{E}$ ). The Jenkins formula is written as, $\mathrm{D}(\mathrm{d})=(\sin \theta / \mathrm{a}+\mathrm{d})^{2}\left\{\mathrm{D}_{1} \mathrm{e}^{-\rho \mathrm{d} / \lambda 1}+\mathrm{D}_{2} \mathrm{e}^{-\mathrm{\rho d} / \lambda 2}+\mathrm{D}_{3} \mathrm{e}^{-\mathrm{\rho d} / \lambda 3}\right\}$
$($ Rem/electron $)$

where $\theta$ is angle from target to measurement point, $d$ is shield thickness $(\mathrm{cm})$, a is target-to-shield distance (cm), $D_{1}, D_{2}, D_{3}$ are initial dose at $\mathrm{d}=0$ for HE, MID, and GR respectively (Rem $\left.\mathrm{cm}^{2} / \mathrm{e}^{-}\right), \rho$ is shield density $\left(\mathrm{g} / \mathrm{cm}^{3}\right)$, and $\lambda_{1}, \lambda_{2}, \lambda_{3}$ are removal mean-free path (attenuation coefficient) for neutrons in shield for HE, MID, and GR respectively.

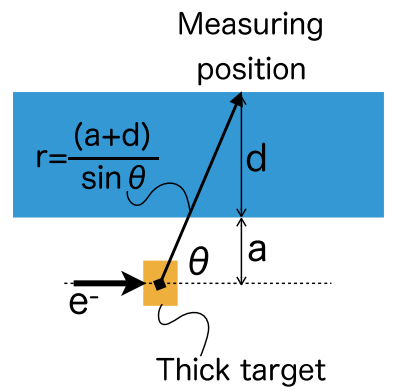

Figure 1. Geometry of the calculation using Jenkins formula. 


\subsubsection{Source term and initial dose}

Neutron yields produced from GeV energy electrons on thick target summarized in Ref. [1] for HE, MID, and GR are shown below respectively.

$$
\begin{aligned}
& \text { HE: } \quad \mathrm{dN} / \mathrm{d} \Omega=1.5 \times 10^{-4} \mathrm{E}_{0} /(1-0.72 \cos \theta)^{2}(\mathrm{n} / \mathrm{sr} / \mathrm{e}-) \\
& \text { MID: } \mathrm{dN} / \mathrm{d} \Omega=3.13 \times 10^{-3} \mathrm{E}_{0} /(1-0.75 \cos \theta)(\mathrm{n} / \mathrm{sr} / \mathrm{e}-) \\
& \text { GR: } \quad \mathrm{Y}=1.49 \times 10^{-2} \mathrm{E}_{0} Z^{0.73}
\end{aligned}
$$

where $E_{0}$ is induced electron energy $(\mathrm{GeV}), \mathrm{Z}$ is atomic number of target, and $\mathrm{Y}$ is total neutron yield.

Fluence-to-dose conversion coefficient used in Ref. [1] are

$$
\begin{array}{lll}
\text { HE: } & 1.15 \times 10^{-7} & (\operatorname{Rem} /(\mathrm{n} / \mathrm{cm} 2)) \\
\text { MID: } & 3.2 \times 10^{-8} & (\operatorname{Rem} /(\mathrm{n} / \mathrm{cm} 2)) \\
\text { GR: } & 3.2 \times 10^{-8} & (\operatorname{Rem} /(\mathrm{n} / \mathrm{cm} 2))
\end{array}
$$

Multiplying the neutron yields by the corresponding conversion coefficient, the initial dose values of $\mathrm{D}_{1}, \mathrm{D}_{2}$, and $\mathrm{D}_{3}$ are obtained as,

$$
\begin{gathered}
\mathrm{D}_{1}=1.5 \times 10^{-4} \mathrm{E}_{0} /(1-0.72 \cos \theta)^{2} \times 1.15 \times 10^{-7} \\
=1.73 \times 10^{-11} \mathrm{E}_{0} /(1-0.72 \cos \theta)^{2} \quad\left(\mathrm{Rem} \mathrm{cm}^{2} / \mathrm{e}^{-}\right) \\
\mathrm{D}_{2}=3.13 \times 10^{-3} \mathrm{E}_{0} /(1-0.75 \cos \theta) \times 3.2 \times 10^{-8} \\
=10.0 \times 10^{-11} \mathrm{E}_{0} /(1-0.75 \cos \theta)\left(\mathrm{Rem} \mathrm{cm}^{2} / \mathrm{e}^{-}\right) \\
\mathrm{D}_{3}=1.49 \times 10^{-2} \mathrm{E}_{0} / 4 / \pi \times 3.2 \times 10^{-8} \\
=3.79 \times 10^{-11} \mathrm{E}_{0} \mathrm{Z}^{0.73}\left(\mathrm{Rem} \mathrm{cm}^{2} / \mathrm{e}^{-}\right)
\end{gathered}
$$

where $\mathrm{D}_{1}$ is in fact revised into $\underline{1.0} \times 10^{-11} \mathrm{E}_{0} /$ $(1-0.72 \cos \theta)^{2}$ when the source term of MID was first included into the formula of older version (i.e., the previous version of Jenkins formula had only HE and GR terms). Considering the modification, the initial dose values can be summarized as

$$
\begin{aligned}
& \text { HE: } \quad D_{1}=1.0 \times 10^{-11} \mathrm{E}_{0} /(1-0.72 \cos \theta)^{2}\left(\mathrm{Rem} \mathrm{cm}^{2} / \mathrm{e}^{-}\right) \\
& \text {MID: } \mathrm{D}_{2}=10.0 \times 10^{-11} \mathrm{E}_{0} /(1-0.75 \cos \theta)\left(\mathrm{Rem} \mathrm{cm}^{2} / \mathrm{e}^{-}\right) \\
& \text {GR: } \quad \mathrm{D}_{3}=3.79 \times 10^{-11} \mathrm{E}_{0} \mathrm{Z}^{0.73} \quad\left(\mathrm{Rem} \mathrm{cm}^{2} / \mathrm{e}^{-}\right)
\end{aligned}
$$

\subsection{Reanalyzing the benchmark data}

The original Jenkins formulae of Eqs. (1) and (5) to (7) are developed based on the measured results carried out at SLAC [2]. In this section of 2.2, the benchmark data is reanalyzed using recent computing codes and data.

\subsubsection{Original benchmark data for Jenkins formula}

Experiments were carried out at SLAC [2] at a collision station where $15 \mathrm{GeV}$ electron beam hit on $8 \mathrm{~cm}$ iron target. Neutron doses were measured outside the station using Rem counter, for different concrete shield thickness from $40 \mathrm{~cm}$ to $200 \mathrm{~cm}$. Since the Rem counter used in the experiment has a response falling sharply down for neutron energy above $5 \mathrm{MeV}$, they assumed higher energy neutrons above $10 \mathrm{MeV}$ could not be detected. In order to compensate the lack of contributions of neutrons above $10 \mathrm{MeV}$, a correction factor was evaluated. It was 0.6 for all measured data, i.e., every measured data was divided by a factor of 0.6 in the analysis [2].

\subsubsection{Monte Carlo calculation}

The factor of 0.6 cannot be constant for different concrete depth, if the shape of the neutron energy spectrum changed during penetration, especially at the beginning of the shield. In order to verify it, Monte Carlo calculation was performed using PHITS [3].

Beam particle and energy, and target material and thickness are set as same as the SLAC experiment. Geometry is a cylindrical concrete tunnel of $200 \mathrm{~cm}$ inner radius as shown as Figure 2. Neutron dose is calculated for 90 degrees in concrete in a detection region of $-50 \mathrm{~cm}<\mathrm{z}<50 \mathrm{~cm}$.

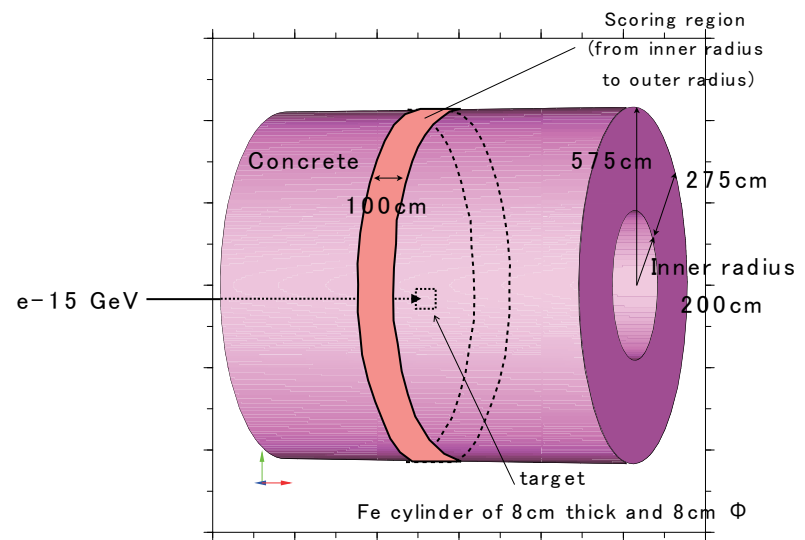

Figure 2. Cylindrical concrete geometry for neutron deep penetration calculation. Inner radius is $200 \mathrm{~cm}$ and thickness of the concrete is $275 \mathrm{~cm}$. At the center an $8 \mathrm{~cm}$ thick iron target locates. Neutron dose is scored in detection region of $-50 \mathrm{~cm}<$ $\mathrm{z}<50 \mathrm{~cm}$ and $200 \mathrm{~cm}<\mathrm{r}<250 \mathrm{~cm}$.

Physical models used in the PHITS calculations are, EGS5[5] for electron and photon shower, the Giant resonance and quasi-deuteron disintegration models for photo-nuclear reaction, and JENDL-HE2007[4] evaluated data library for neutron transport.

The analysis was done as follows. Ratios of neutron dose below $10 \mathrm{MeV}$ to whole energies, i.e., "NDOSE(0-10MeV) / NDOSE(total)", at different depth of concrete position were calculated. It becomes the correction factor for the SLAC experiment. In this evaluation, the limit of detection efficiency of 10 $\mathrm{MeV}$ is just applied (detector response evaluation of the Rem counter is not discussed).

Figure 3 shows the result. The original correction factor evaluated at SLAC of 0.6 is certainly valid at thick concrete positions (above $100 \mathrm{~cm}$ ). On the other hand, it becomes smaller at thinner concrete thickness. For example, it is a value of about 0.8 at concrete thickness of $50 \mathrm{~cm}$. The new correction factors as a function of concrete thickness will be applied to measured data instead of the constant correction factor of 0.6 .

In the calculation, density of concrete was set to 2.26 $\left(\mathrm{g} / \mathrm{cm}^{3}\right)$ as described in Ref. [1]. It is noted that the neutron dose deep penetration calculation result is rather sensitive to concrete composition (especially amount of 
Hydrogen). Several ordinary concrete compositions are chosen from Ref. [6] and compared to the experimental data as shown later (in Figure 4). The Los Alamos concrete composition is selected for all calculations in this study since the simulated result agrees best to the SLAC experimental data. The Hydrogen atom fraction in concrete, which can differ at every production district or environmental conditions during use, becomes an additional key parameter (i.e., thickness, density, and $\mathrm{H}$ content of concrete) to evaluate neutron deep penetration. The values were NIST 0.31 , US03 0.15, and Los Alamos 0.085 in Ref. [6].

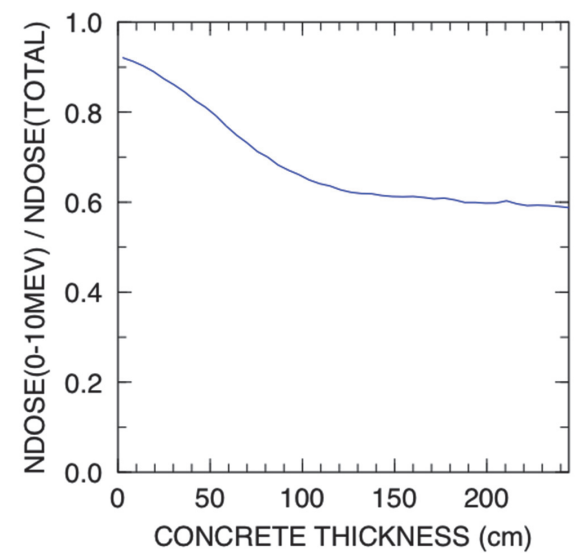

Figure 3. New correction factor to SLAC experiment (Ratio of neutron dose below $10 \mathrm{MeV}$ to neutron dose for whole energies) as a function of concrete thickness.

Table 1. Concrete composition used in PHITS.

\begin{tabular}{cc}
\hline Target nucleus & $\begin{array}{c}\text { Atom fraction } \\
\text { (No.86 in ref[6]) }\end{array}$ \\
\hline${ }^{1} \mathrm{H}$ & 0.08474 \\
${ }^{16} \mathrm{O}$ & 0.6041 \\
${ }^{23} \mathrm{Na}$ & 0.01252 \\
${ }^{27} \mathrm{Al}$ & 0.02484 \\
${ }^{\mathrm{nat}} \mathrm{Si}$ & 0.2419 \\
${ }^{\mathrm{nat}} \mathrm{Ca}$ & 0.02724 \\
${ }^{n a t} \mathrm{Fe}$ & 0.004652 \\
\hline
\end{tabular}

\subsubsection{Reanalyzing the benchmark data}

Using the new correction factors discussed in the previous section, the benchmark data were reanalyzed. The result is shown in Figure 4 together with PHITS calculations. It is noted that the concrete shield begins at $200 \mathrm{~cm}$ in Figure 4, i.e., " $\mathrm{x}=200$ in Figure 3 (concrete $200 \mathrm{~cm}$ ) corresponds to $\mathrm{x}=400$ in Figure 4 (distance $400 \mathrm{~cm}$ from target)". Compared to the original benchmark data (filled square), the reanalyzed benchmark data (open circle) shows smaller neutron dose for the concrete thickness less than $100 \mathrm{~cm}$ (due to the higher correction factors).

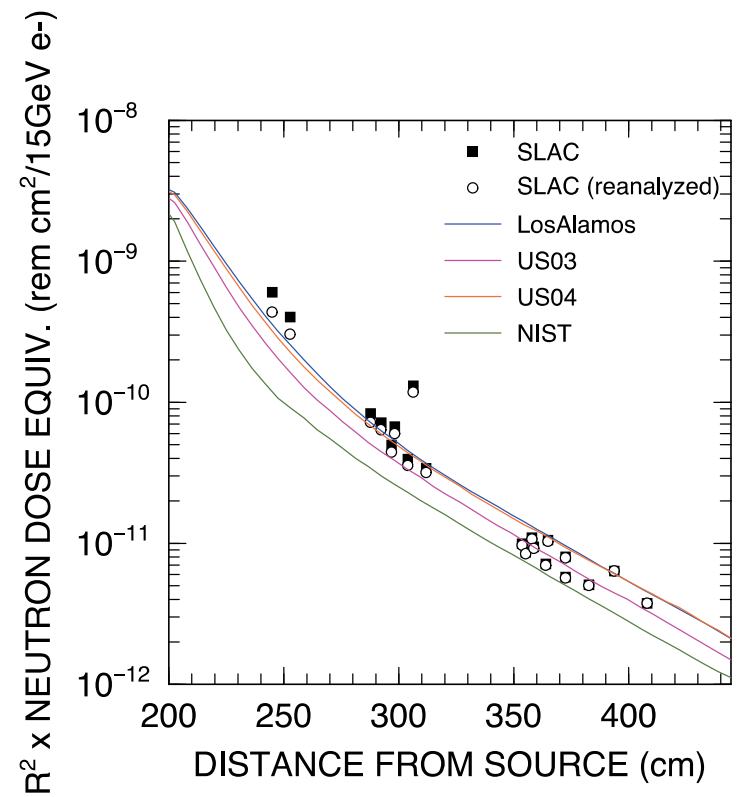

Figure 4. The original and reanalyzed SLAC data, and neutron attenuation calculation for various ordinary concrete for 90 -degree direction $15 \mathrm{GeV}$ electron on iron target.

\subsection{Re-parametrization of the formula}

The parameters of the original Jenkins formula were decided so as the total neutron attenuation curve fits best to the benchmark data. Since the benchmark data was revised, the parameter sets of the formula should be also revised.

Figure 5 shows the comparison of the original Jenkins formula, SLAC experiment (original and reanalyzed one), and Monte Carlo simulation.

In Figure 5, the three components are also plotted together with PHITS simulation results, and good agreement is obtained overall on both the initial value (source term) and the gradient of the curves (attenuation coefficient). It can be indicated that the concept of Jenkins formula is actually correct from the analysis using the recent computing codes and data libraries in this study. On the other hand, there are certain amount of discrepancies can be seen in some parameters. Table 2 shows the comparison of the attenuation coefficients of the three components. Especially for MID energy, discrepancy is seen. In addition, in Figure 5, on the initial values at $200 \mathrm{~cm}$, which represent the initial dose of $D_{1}, D_{2}, D_{3}$ (the source term) of the formula, the solid line of MID ( $\mathrm{D}_{2}$ of the original Jenkins) and the dashed line of MID ( $\mathrm{D}_{2}$ of PHITS) differs. It is pointed out that at least the two parameters of $\lambda_{2}$ and $D_{2}$ should be revised in this work. 


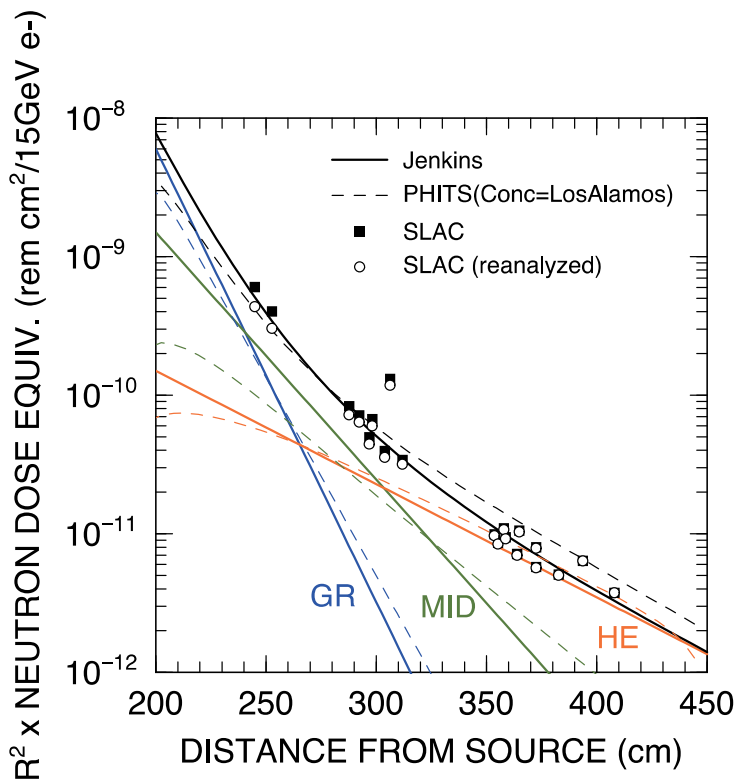

Figure 5. Comparison between the original benchmark data, reanalyzed benchmark data, original Jenkins formula (total neutron dose and each energy groups of GR, MID, HE), and PHITS (same as Jenkins).

Table 2. Neutron dose attenuation coefficient of Jenkins formula and PHITS simulation.

\begin{tabular}{lcc}
\hline $\begin{array}{l}\text { Energy group } \\
\text { (representativ } \\
\text { e value })\end{array}$ & $\begin{array}{c}\lambda \\
\text { Jenkins } \\
\left(\mathrm{g} / \mathrm{cm}^{2}\right)\end{array}$ & $\begin{array}{c}\lambda \\
\text { PHITS } \\
\left(\mathrm{g} / \mathrm{cm}^{2}\right)\end{array}$ \\
\hline GR $(10 \mathrm{MeV})$ & 30 & 35 \\
MID $(75 \mathrm{MeV})$ & 55 & 75 \\
HE $(200 \mathrm{MeV})$ & 120 & 126 \\
\hline
\end{tabular}

\section{Results - a revised Jenkins formula}

The validity of the concept of Jenkins formula, its attenuation coefficients, and the initial values of the attenuation curves is proved by the evaluations. On the other hand, it is also pointed out that the measured data can be revised a small amount at thin concrete positions, and also some parameters used in Jenkins formula can be renewed if new fitting analysis is applied to the revised measured data. The key parameters are both the attenuation coefficient $\lambda_{2}$ and the source term $D_{2}$ for MID neutrons as a significant discrepancy can be seen between original and calculation in Figure 5.

New parameters for Jenkins formula are determined by following procedures, (1) the attenuation coefficient $\lambda_{1}, \lambda_{2}, \lambda_{3}$ are replaced to which obtained by PHITS results shown in Table 2, and (2) new fitting analysis is performed with the condition that the source term of MID neutrons $\mathrm{D}_{2}$ is an unknown parameter. As a result, $\mathrm{D}_{2}$ is obtained as $1.32 \times 10^{-11} \mathrm{E}_{0} /(1-0.75 \cos \theta)$ instead of $10 \times 10^{-11} \mathrm{E}_{0} /(1-0.75 \cos \theta)$. Finally, the revised Jenkins formula can be described as,

$\mathrm{D}(\mathrm{d})=\mathrm{E}_{0} \times 10^{-11}(\sin \theta / \mathrm{a}+\mathrm{d})^{2}\left\{\mathrm{e}^{-\mathrm{pd} / 35}+1.32 /(1-0.75 \cos \theta)\right.$ $\left.\mathrm{e}^{-\rho \mathrm{d} / 75}+3.79 \times 10^{-11} \mathrm{Z}^{0.73} \mathrm{e}^{-\rho \mathrm{d} / 126}\right\}$ (Rem/electron)
The comparison between original formula, revised formula, and the new benchmark data is shown in Figure 6. A revised Jenkins formula was plotted by dashed line, which represents the reanalyzed benchmark data better than the original formula. Revised formula shows higher value than the original for concrete thickness thicker than $150 \mathrm{~cm}$, because the attenuation coefficients for higher energy (especially $\lambda_{2}, \lambda_{3}$ ) become larger, on the other hand, revised formula gives slightly lower value than the original for thinner concrete shield of $150 \mathrm{~cm}$.

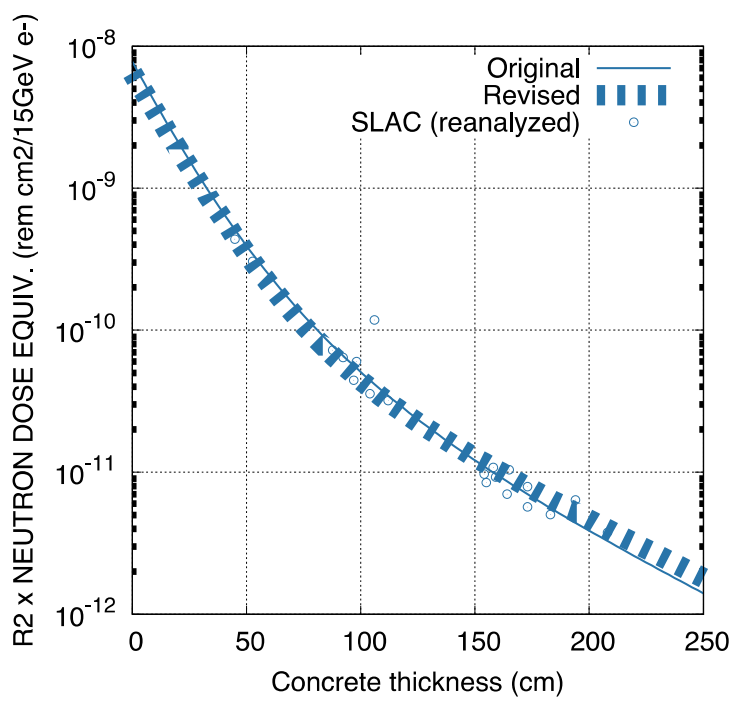

Figure 6. A revised Jenkins formula, which obtained by fitting the reanalyzed benchmark data compared to the original.

\section{Discussion}

\section{1. $\lambda$ values}

The attenuation coefficients $\lambda_{1}, \lambda_{2}$, and $\lambda_{3}$ in the revised Jenkins formula are larger than ones of the original. As a result, the revised formula gives higher neutron dose at concrete depth thicker than $150 \mathrm{~cm}$. Validation of new attenuation coefficients should be performed in future, but there is a reason that the revised $\lambda_{1}, \lambda_{2}$, and $\lambda_{3}$ may not differ much from correct values; it is because $\lambda$ is determined by cross sections of concrete elements and an equilibrium neutron energy spectrum (the shape of the equilibrium spectrum is actually decided also by cross sections), and in this study the JENDL-HE2007 evaluated cross sections are used, which is quite reliable and it is hard to find better theoretical methods at present.

\subsection{Electron induced neutron production source terms}

\subsubsection{Comparison}

Concerning the accuracy of the source term of electron induced neutron production, there may be a largest uncertainty in this study. Comparison between Jenkins source terms of GR, MID, HE neutrons and PHITS results is shown in Figure 7. Overall satisfactorily agreements are obtained. It is noted that in 
the comparison, the Jenkins source terms of Eq. (2), (3) and (4) are plotted after dividing by factors of "pseudo source factor" as explained in the next section of 4.2.2.

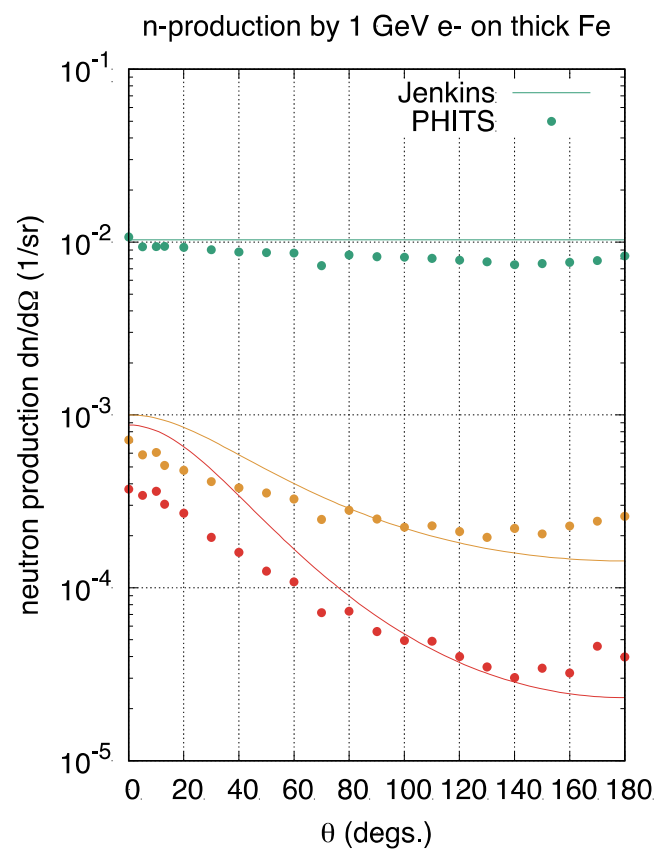

Figure 7. Comparison between Jenkins source terms of GR (colored by green), MID (orange), HE (red) neutrons and PHITS calculation. It is noted that the Jenkins source terms of GR, MID, and HE are divided by 1.24, 1.65, and 2.19 respectively, for invalidating the pseudo source factor, which is described in Sec. 4.2.2.

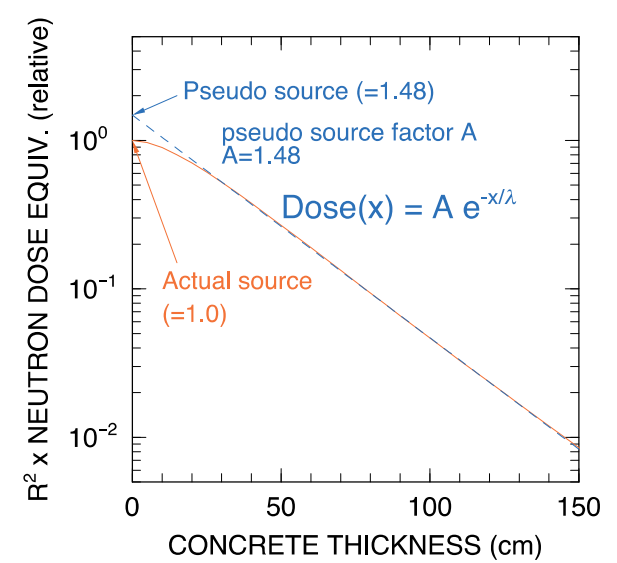

Figure 8. Schematic plot of high energy neutron attenuation in concrete.

\subsubsection{Pseudo and actual source values in Jenkins formula}

It is well known that the Buildup takes place at the beginning of shield in radiation attenuation in matter. Typical case of neutron attenuation in concrete is shown in Figure 8. The initial dose at shield thickness $0 \mathrm{~cm}$ is normalized to 1 . When the attenuation curve is fitted by a simple exponential function for the straight-line part (dashed line), the extrapolated value at shield thickness $0 \mathrm{~cm}$ reaches not to the original value of 1 but higher (in Figure 8 it is 1.48). It is of course due to results of Buildup. The Buildup reaction should occur also in the
SLAC experiment. How the Buildup term is included in Jenkins formula is an interesting question. Here we define the initial source value at shield $0 \mathrm{~cm}$ (the value is 1 in Figure 8) as "actual source" and the extrapolated values (of 1.48 in Figure 8 ) as "pseudo source". In Jenkins formula, the source terms $\mathrm{D}_{1}, \mathrm{D}_{2}$ and $\mathrm{D}_{3}$ are described actually by "pseudo source" of each energy groups. The ratio of pseudo source to actual source can be called as "pseudo source factor", and in case of Jenkins formula, the factors are 1.24, 1.65, and 2.19 for GR, MID, and HE neutrons respectively by calculation of PHITS using the same geometry of Figure 2. The calculations were performed using the mono energy neutron source of representative values of 10, 75, and $200 \mathrm{MeV}$ for GR, MID and HE respectively.

\section{Conclusion}

The validity of the concept of Jenkins formula is proved in this study using evaluations of recent Monte Carlo calculation. It is remarkable that the formula represents neutron deep penetration in concrete with quite sufficient accuracy. The benchmark data can also be reanalyzed with supports of the modern computing codes and evaluated libraries. By performing a new fitting analysis for the reanalyzed benchmark data, it is indicated that several parameters of the formula can be revised as described as Eq.5, which will give higher dose than the original at concrete thickness deeper than $150 \mathrm{~cm}$ even the difference is not large from the original.

\section{References}

[1] T.M. Jenkins, Neutron and photon measurements through concrete from a $15 \mathrm{HeV}$ electron beam on a target - comparison with models and calculations, Nuclear Instruments and Methods 159 (1979), pp. 265-288.

[2] W. Ash et al., SLAC TN-77-5 (1977).

[3] T. Sato, K. Niita, N. Matsuda, S. Hashimoto, Y. Iwamoto, S. Noda, T. Ogawa, H. Iwase, H. Nakashima, T. Fukahori, K. Okumura, T. Kai, S. Chiba, T. Furuta and L. Sihver, Particle and heavy ion transport code system PHITS, Version 2.52, J. Nucl. Sci. Technol. 50 (2013), pp. 913-923.

Y. Iwamoto, T. Sato, S. Hashimoto, T. Ogawa, T. Furuta, S. Abe, T. Kai, N. Matsuda, R. Hosoyamada and K. Niita, Benchmark study of the recent version of the PHITS code, J. Nucl. Sci. Technol. 54 (2017), pp. 617-635.

[4] T. Saga, T. Sugawara, K. Kosako and T. Fukahori, Continuous energy cross section library for MCNP/MCNPX based on JENDL High Energy File 2007, JAEAI-Data/Code 2008-022, Japan Atomic Energy Agency, (2008).

[5] H. Hirayama, Y. Namito, A.F. Bielajew, S.J. Wilderman and W.R. Nelson, SLAC-R-730 (2005) and KEK Report 2005-8 (2005).

[6] R.J. McConn Jr. et al., Compendium of material composition data for radiation transport modeling, PIET-43741-TM-963, PNNL-15870 Rev. 1 